\title{
Interstitial muons and hydrogen in diamond and silicon
}

\author{
Alison Mainwood $\dagger$ and A M Stoneham \\ Theoretical Physics Division, AERE Harwell, Oxon OX11 ORA, UK
}

Received 8 November 1983

\begin{abstract}
We have calculated self-consistent total-energy surfaces for hydrogen present interstitially as $\mathrm{H}^{+}, \mathrm{H}^{0}$ and $\mathrm{H}_{2}$ in crystalline silicon and diamond. The dissimilarities of the two materials are more evident than their similarities, for we show molecular hydrogen to be the stable form in silicon, and atomic hydrogen to be the stable form in diamond in the absence of impurities. The energy surfaces for $\mathrm{H}^{0}$ and $\mathrm{H}^{+}$are complex, with minima too small to trap the atoms when zero-point energy is taken into account. We discuss our results in relation to other theories and to the normal and anomalous muonium $\left[\mu^{-} \mathrm{e}^{-}\right]$experiments.
\end{abstract}

\section{Introduction: three fundamental puzzles}

Hydrogen in amorphous silicon has been studied to an enormous extent. In the crystalline semiconductors there has been a much smaller effort, which has concentrated more closely on technical studies of phenomena like electrical isolation (see, e.g., Steeples et al 1980). Recently, three groups of experimental results have called for a reassessment of the scientific and practical importance of hydrogen in crystalline materials: the tunnelling states in ultra-pure $\mathrm{Ge}$, the muon-spin-rotation experiments on diamond, $\mathrm{Si}$ and $\mathrm{Ge}$ which showed many unexpected (and currently unexplained) features, and the realisation that hydrogen was plentiful in diamond, not absent as often believed.

Any useful theory of hydrogen in group IV crystals (diamond, silicon and germanium) must explain three fundamental observed features $\ddagger$. First, why is hydrogen inactive electrically and optically? Even though large hydrogen concentrations are reported in diamond, and even though weak features associated with hydrogen are seen, it is clear that most hydrogen is in an unidentified state unassociated with these features. Secondly, muon spin rotation identifies two quite distinct states of muonium (the analogue $\left[\mu^{+}\right.$e] of neutral hydrogen): normal muonium, with a compact electronic wavefunction with mainly s character at the muon, and anomalous muonium, with (111) symmetry and only a weak electronic amplitude at the muon. Whilst there are some doubts about whether these states are both intrinsic, and whilst there is the possibility that more complex states (e.g. $\left[\mu^{+}\right.$e eh]) could be involved, the universality of the behaviour suggests that any impurities only play a secondary role in determining the nature of the system. Thirdly, pion experiments (essentially $\mu^{+}$channelling from the

† Present address: Department of Physics, King's College, London WC2R 2LS, UK.

¥ To avoid complicating the text, a fuller survey and analysis of the experimental position and references are given in a separate Appendix. 
decay of a localised pion) indicate a change of the $\pi^{+}$site in Ge from an off-centre position $(T \leqslant 60 \mathrm{~K})$ to on-centre in a tetrahedral site $(60 \mathrm{~K} \leqslant T \leqslant 80 \mathrm{~K})$. Which states are involved? If, having explained these features, the theory also agreed with the related work on hydrogen (probably associated with a damage centre) in implanted silicon and on hydrogen tunnelling in $\mathrm{Ge}$, so much the better.

Current theories fall far short of answering (and often of recognising) these issues. The main reason is that, with very rare exceptions, theories of hydrogen impurities concentrate on the one-electron levels for hydrogen presumed to be at the tetrahedral site (Rodriguez et al 1979, Wang and Kittel 1973, Herbert and Inkson 1977, Altarelli and Hsu 1979). The weakness of such approaches, whether by extensions of effectivemass theory (Singh et al 1977, 1978, Coker et al 1980) or by non-self-consistent molecular orbital theories (Pickett and Cohen 1980), is that they do not calculate the total-energy surfaces on which most observed features depend.

\section{The cNDo molecular orbital method}

\subsection{General features}

The CNDO (complete neglect of differential overlap) method was devised mainly with organic molecules in mind, and has been quite successful for them (Pople and Beveridge 1970). It is a semi-empirical method and can be easily applied and parametrised while retaining the physical sense of the application.

It starts from the Hartree-Fock-Roothaan equations, neglects terms of the order of the overlap between orbitals on different atoms, and approximates other matrix elements systematically. A basis set of Slater orbitals is used on the outer electrons only, in our case $1 \mathrm{~s}$ for $\mathrm{H}, 2 \mathrm{~s}, 2 \mathrm{p}$ for $\mathrm{C}, 3 \mathrm{~s}, 3 \mathrm{p}$ for $\mathrm{Si}$. Three types of parameter are used:

(i) orbital exponents, $\zeta$, which determine overlaps in expressions for diagonal matrix elements and the Coulomb integrals used to evaluate electron-electron integrals;

(ii) electronegativities, $\varepsilon_{\mathrm{s}}, \varepsilon_{\mathrm{p}}$, which determine the relative attraction of the electrons for the different orbitals;

(iii) bonding parameters $\beta$, which fix the degree of bonding through the resonance integrals.

The CNDO program gives self-consistent solutions, similar to Hartree-Fock solutions. These comprise one-electron energy levels, the total energy, the wavefunctions and a measure of the charge associated with each atom. The total energy calculated by this method is not simply a sum of one-electron energies, as is the case in some other similar schemes, but is calculated to account correctly for the electron-electron repulsion. Therefore the total energies obtained from a series of calculations can be used to build up a realistic potential energy surface of the hydrogen in the lattice. The total energies can also show how lattice relaxation affects behaviour.

\subsection{Choice of empirical parameters}

The parameters (table 1) for these semi-empirical calculations are determined entirely independently from the defect calculations to be described here. The defect calculations are thus fully defined, i.e. we have no adjustable parameters, nor do we add in any further experimental data (such as band gaps) which some approaches allow. The 
parameters for carbon and silicon were obtained by Harker and Larkins (1979). They treated the parameters as adjustable, starting initially from exponents and electronegativities close to atomic values. Using a periodic cluster of 18 atoms in a diamond or silicon lattice, they altered the parameters systematically until they obtained a best fit to the experimental values of equilibrium spacing, cohesive energy and the width and approximate structure of the valence band. The parameters used for hydrogen were those given by Pople and Beveridge since, in conjunction with the Harker and Larkins carbon parameters, they reproduced well the bond lengths and energies for some small organic molecules such as $\mathrm{CH}_{4}$ and $\mathrm{C}_{2} \mathrm{H}_{6}$.

Table 1. CNDO parameters.

\begin{tabular}{llccc}
\hline & $\begin{array}{l}\text { Orbital exponents } \\
\zeta\left(\mathrm{au}^{-1}\right)\end{array}$ & $\begin{array}{l}\text { Bonding parameters } \\
\beta(\mathrm{eV})\end{array}$ & \multicolumn{2}{c}{$\begin{array}{c}\text { Electronegativities } \\
\varepsilon_{\mathrm{s}}(\mathrm{eV})\end{array}$} \\
\hline $\mathrm{H}$ & 1.2 & -9.0 & 7.18 & - \\
$\mathrm{C}$ & 1.765 & -10.2 & 7.0 & 5.5 \\
$\mathrm{Si}$ & 1.54 & -6.4 & 6.3 & 4.5 \\
$\mathrm{C}^{*}$ & 1.8 & 0 & 7.0 & 5.5 \\
$\mathrm{Si}^{*}$ & 1.54 & 0 & 6.3 & 4.5 \\
\hline
\end{tabular}

At the edges of the clusters, the carbon and silicon atoms denoted $\mathrm{C}^{*}$ and $\mathrm{Si}^{*}$ (this is explained below) had slightly different parameters, since it was essential to get convergence of the calculations and to suppress a tendency for all the electrons to crowd into the orbitals around the surface of the cluster.

The CNDO method and the Harker-Larkins parametrisation have proved to be very satisfactory in a number of applications - the vacancy (Mainwood 1978), self-interstitials (Mainwood et al 1978) and substitutional atoms (Mainwood 1979) in diamond and the silicon self-interstitial (Masri et al 1983) -where this theory reproduced experimental results in all cases.

\subsection{Cluster size and shape}

The main constraint in the choice of clusters is the computer core storage required. It is necessary to diagonalise a matrix of the order of the number of basis orbitals in the cluster, a procedure that uses a great deal of space and time. We therefore chose clusters containing about 70 atoms and, to economise on space, represented each of those atoms on the edges of the cluster only by the $\mathrm{sp}^{3}$ hybrid orbital that bonded it to the rest of the cluster. These partial atoms are the ones we have denoted $\mathrm{C}^{*}$ and $\mathrm{Si}^{*}$.

Most of the calculations were done on a 70-atom cluster (figure 1) (30Si $40 \mathrm{Si}^{*}$ or $30 \mathrm{C}$ $40 \mathrm{C}^{*}$ ) centred on a tetrahedral interstitial site and including the seven nearest shells of atoms. A few further calculations were made on 59 -atom clusters (29Si $20 \mathrm{Si}^{*}$ ) to ensure both that the effects of the edges of the cluster were not substantial and that the choice of the cluster's central site was not critical. This 59-atom cluster is centred on a substitutional site and contains shells of atoms up to its sixth neighbours.

Since investigation showed that only very small lattice relaxations were necessary in these calculations, only the nearest neighbours of the hydrogen defect have been relaxed. Large lattice relaxations would obviously require further atoms to be displaced too, as in some of our previous calculations for other defect systems. 


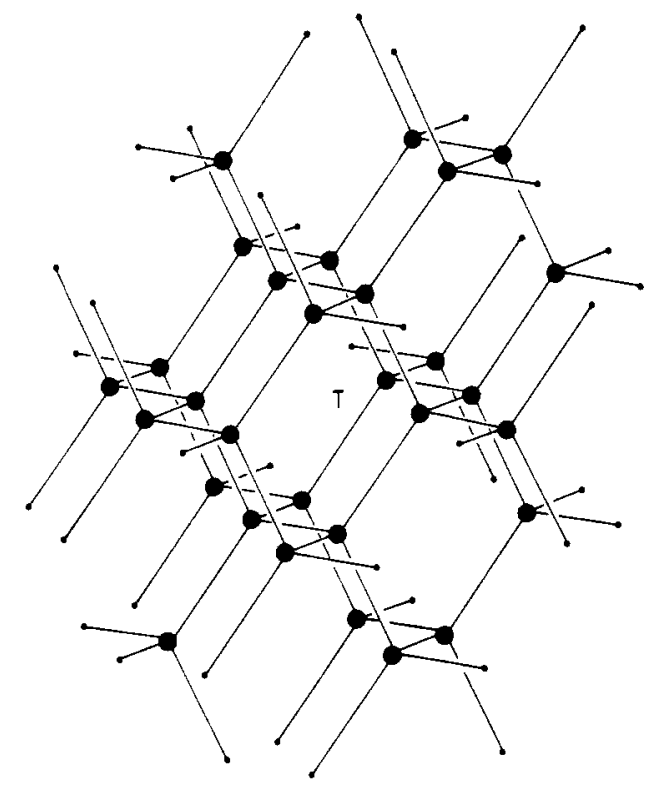

Figure 1. The 70 -site cluster used in most calculations. The central tetrahedral site is labelled $\mathrm{T}$. Large full circles represent $\mathrm{Si}$ atoms, and smaller full circles the $\mathrm{Si}^{*}$ surface-saturating silicon bonds.

\subsection{Zero-point motion}

Since the muon, $\mu^{+}$, is chemically like a very light proton, CNDO calculations on a potential surface of hydrogen in a lattice also give adiabatic energy surfaces for muonium. The difference in the masses does not affect the relative potential energies of the sites for $\mathrm{H}$ and $\mathrm{Mu}$, but does drastically affect the zero-point energy and does alter the other vibrational energy levels which the hydrogen or muonium can inhabit. We shall give estimates of the zero-point energy in due course.

\subsection{Charge states and other excitations}

We have used two charge states for the total cluster: positive, which would correspond to $\mathrm{H}^{+}$or $\mu^{+}$in the lattice, and neutral for $\mathrm{H}^{0}$ or $\mathrm{Mu}\left(\mu^{+} \mathrm{e}^{-}\right)$. However, because of the self-consistent nature of the calculations, it is not possible to impose localisation of the charge on the hydrogen itself. In the case of silicon we find the $\mathrm{H}^{+}$and $\mathrm{H}$ states are quite distinct. In the case of $\mathrm{H}^{+}$(or $\mu^{+}$) in diamond, however, the results (including the effective charge on the hydrogen) were identical with $\mathrm{H}\left(\right.$ or $\mu^{+} \mathrm{e}^{-}$). Here the proton or muon had captured an electron that should have been bound in an orbital on the surface of the cluster. We infer that the capture of an electron will be very rapid and there might not be a stable $\mathrm{H}^{+}$(or $\mu^{+}$) state. The conclusion is not clear-cut, however, for the reaction transferring an electron from a neutral hydrogen-free cluster to a positively charged cluster containing hydrogen is endothermic. This suggests there is an energy cost in moving an electron from the valence band to the $\mathrm{H}^{+}$.

Further electronic states of the muon (perhaps accounting for the anomalous form) would include trapped-exciton states like $\mu^{+} e^{-} e^{-} h^{+}$. We are unable to explore states like this using CNDO because they do not converge. 


\section{Energy surfaces for $\mathbf{H}^{+}$and $\mathrm{H}^{0}$ in diamond and silicon}

Almost all the interesting interstitial sites can be seen from figure 2, which shows a section through the basic cube of the diamond lattice in a $\langle 110\rangle$ plane. This includes all the high-symmetry features of the interstitial positions, and contains the tetrahedral and
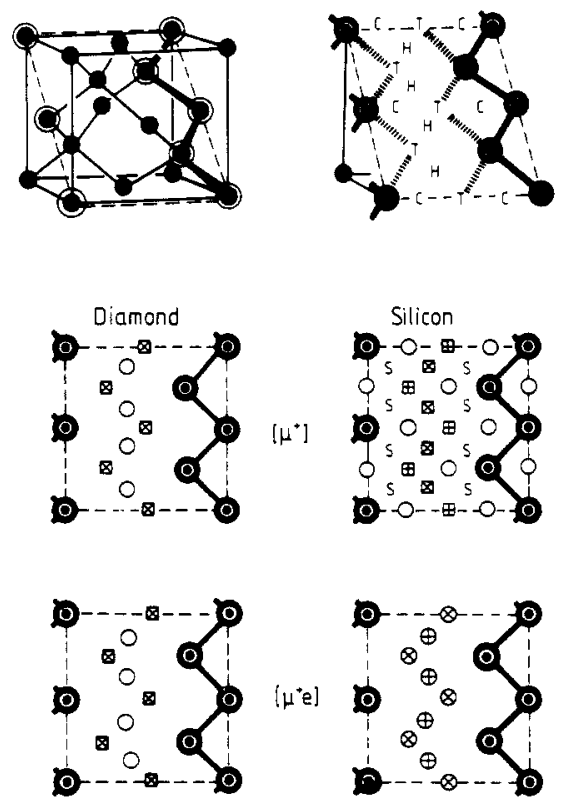

Figure 2. Interstitial sites in the diamond lattice. This diagram also summarises the results of our calculations. $\mathrm{T} \equiv$ tetrahedral site, $\mathrm{H} \equiv$ hexagonal site, $\mathrm{C} \equiv$ site on the tube axis from a lattice site; bonds are shown as full lines and back bonds as broken lines. Circles represent energy minima ( $x$ and + are weak minina, sensitive to geometry) and squares energy maxima; $s$ denotes saddle points.

hexagonal interstitial sites, the line between the tetrahedral site and a nearest-neighbour atom-which constitutes the 'back bond'-and a pair of the next-nearest-neighbour host atoms. For the most part we have used this plane to construct the energy surfaces, but we have examined other planes near the energy minima to find the shape of any apparent potential well more fully in three dimensions.

\section{1. $H^{+}$and $H^{0}$ in diamond}

As explained in $\S 2$, it was not possible to obtain separate $\mathrm{H}$ and $\mathrm{H}^{+}$energy surfaces in diamond. This may mean simply that $\mathrm{H}^{+}$will capture an electron rapidly, and it undoubtedly indicates a need for caution in interpretation.

Figure 3 shows the energy contours of a hydrogen on the [110] plane in diamond. The energy surface is relatively flat, so questions of the degree of accuracy cannot be avoided. There is a local maximum at the tetrahedral site $(\mathrm{T} ; 2.80 \mathrm{eV})$ and a minimum at the hexagonal site $(\mathrm{H} ; 2.21 \mathrm{eV})$. There are saddle points between hexagonal sites along $\langle 001\rangle$ directions from tetrahedral sites $(\mathrm{s} ; 2.33 \mathrm{eV})$. The minimum at the hexagonal site actually dips $8 \mathrm{meV}$ lower in a small ring around the site in the plane of the hexagon. 


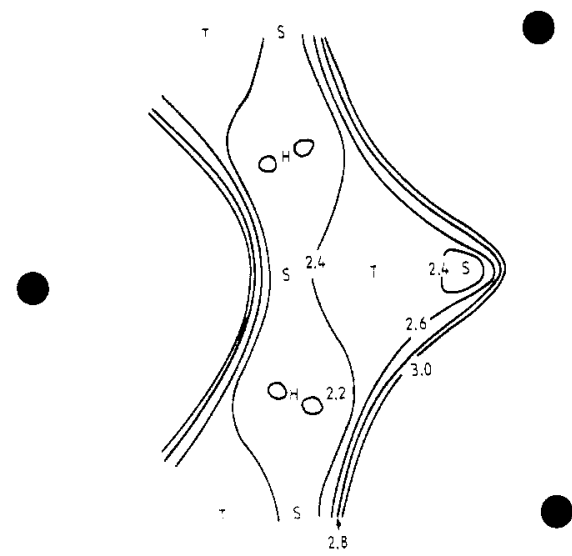

Figure 3. Energy contours in the (110) plane for hydrogen in diamond. The contours are in $\mathrm{eV}$, with energies $2.797 \mathrm{eV}$ (T site), $2.33 \mathrm{eV}$ (saddlepoint s) and $2.205 \mathrm{eV}$ (H site).

Some of these features are too small for any current methods to predict with certainty, and we would not claim that these details are accurately given. Nevertheless, the past successes of CNDO calculations suggest we should treat the qualitative features seriously.

A simple variational calculation shows that the zero-point energy of a proton in the hexagonal minimum would be about $170 \mathrm{meV}$ (slightly lower by the flattening of the well near the saddle points). Hydrogen will therefore not be trapped at such a site, and muonium will certainly not. Deuterium, with a zero-point energy of less than $125 \mathrm{meV}$, may have one localised level.

\section{2. $H^{0}$ in silicon}

Here the hydrogen has an almost spherical potential well centred on the tetrahedral site, but with a narrow low-energy channel via the hexagonal site to the next tetrahedral site (figure 4). The difference in energy between tetrahedral and hexagonal sites is only $0.052 \mathrm{eV}$.

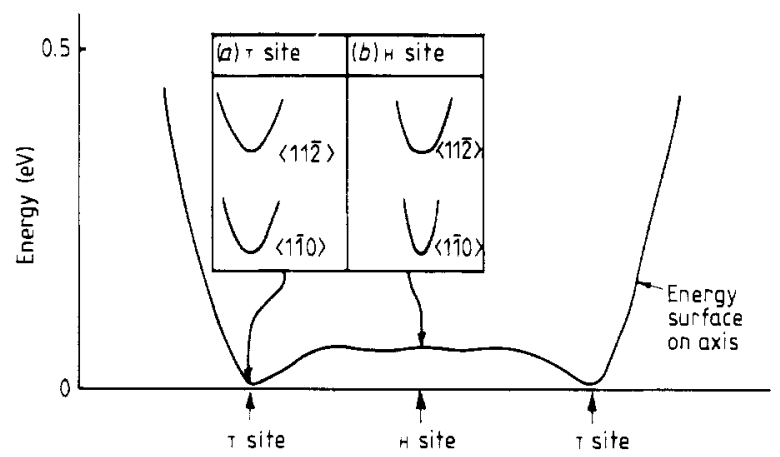

Figure 4. Energy surfaces for $\mathrm{H}^{0}$ in silicon along and close to the T-site-T-site $\langle 111$ ) axis. Lattice relaxation (not shown) would lower the energy at the hexagonal ( $\mathrm{H}$ ) site to almost exactly that at the $\mathrm{I}$ site. The extra curves show how the energy varies as the $\mathrm{H}^{0}$ is moved off the axis in the $\langle 11 \overline{2}\rangle$ and $\langle 1 \overline{1} 0\rangle$ directions. The T-T distance is the Si-Si spacing, i.e. $2.35 \AA$. The diagrams under $(a)$ and $(b)$ indicate the energy surfaces normal to the $\langle 111\rangle$ axis at the site indicated. 
The relaxation of host atoms near the hydrogen alters this picture however. There is very little change of energy for the hydrogen in the tetrahedral site if the four neighbouring silicon atoms are moved, but if the six silicon atoms adjacent to the hexagonal site relax outwards by only $1 \%$ of a lattice spacing, the energy of the hydrogen in the hexagonal position drops to a very similar energy to the tetrahedral one. Localised sites for $\mathrm{D}^{0}, \mathrm{H}^{0}$ and $\mathrm{Mu}$ in silicon thus seems unlikely.

\section{3. $\mathrm{H}^{+}$in silicon}

This energy surface is both quite dissimilar from and more complex than the former two cases. The tetrahedral site is a local maximum, and the hexagonal site is also fairly high in energy (figure 5). There is a minimum along the back-bond directions at about $1.55 \AA$ from a silicon atom but this proves to be a saddle-point only. The minimum along $\langle 001\rangle$ directions is $1.1 \mathrm{eV}$ below the saddle-point and is about $1.6 \AA$ from the silicon atom. This demonstrates the importance of checking low-symmetry sites in defect calculations. The actual minima are either side of this $\langle 001\rangle$ line in the plane of the three nearest silicon atoms and these minima are another $7 \mathrm{meV}$ lower in energy. A direct route between two of the minima of these neighbours associated with different tetrahedral sites surmounts an energy barrier of $0.21 \mathrm{eV}$. We have used a simple variational model to find the zero-point energy of hydrogen in this very anharmonic well, and get an upper limit of $0.22 \mathrm{eV}$. We conclude that hydrogen may just be trapped at this site; deuterium is very likely to have one trapped state, but muonium will not be trapped (zero-point energies $E_{0}^{\mathrm{D}} \geqslant 0.17 \mathrm{eV}, E_{0}^{\mu^{+}} \geqslant 0.57 \mathrm{eV}$ ).

\section{4. $\mathrm{H}_{2}$ in diamond and silicon}

In both diamond and silicon, we find that molecular hydrogen appears to adopt a spacing close to its free space $\mathrm{H}-\mathrm{H}$ distance, and to prefer a $\langle 001\rangle$ orientation in a tetrahedral site. The barrier to molecular rotation is about $43 \mathrm{meV}$ in diamond and $93 \mathrm{meV}$ in silicon. The molecule can migrate from site to site via hexagonal sites, which constitute a barrier of only $0.37 \mathrm{eV}$ in diamond but $0.95 \mathrm{eV}$ in silicon, although both these figures

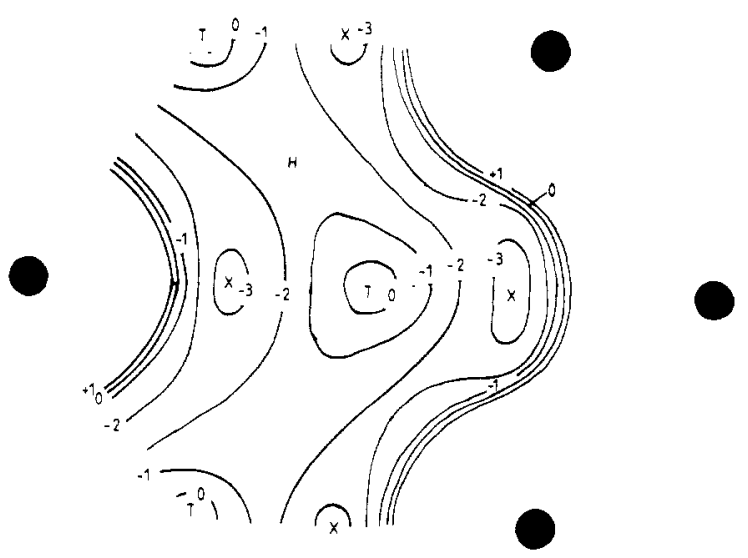

Figure 5. Energy contours for $\mathrm{H}^{+}$in silicon in the (110) plane. The contours are in $\mathrm{eV}$, with energies $0.39 \mathrm{eV}$ ( $\mathrm{T}$ site), $-1.48 \mathrm{eV}$ ( $\mathrm{H}$ site) and $-3.23 \mathrm{eV}$ at the $\mathrm{X}$ site, situated along $\langle 001\rangle$ axes from the tetrahedral site. 
should be reduced by relaxing the lattice around the hexagonal site. As we have noted before (Mainwood and Stoneham 1983), the activation energy for $\mathrm{H}_{2}$ in Si compares favourably with experimental values quoted for hydrogen in silicon.

One important question refers to the relative stability of the atomic molecular forms. In $\mathrm{Si}$ our calculations show $\mathrm{H}_{2}$ is favoured by other $2 \mathrm{eV}$. In diamond, the balance is much closer. Our calculations suggest the atomic form is stable, though by a mere $0.14 \mathrm{eV}$. Molecular hydrogen may still be important at elevated temperatures or, more probably, in association with the many defect centres like the ubiquitous and varied complex nitrogen centres.

\subsection{Comparison with other theoretical work}

Although our conclusions are very different from those of most previous workers, the results on which they are based are often in reasonable accord. Thus, if we constrain hydrogen at the tetrahedral site, the charge density is broadly in line with that given by other workers. If we look at only a small range of geometries, we often find features qualitatively similar to those suggested by others. The main differences we note came from three factors. Firstly, we have looked at many more geometries in defining energy surfaces. Secondly, we have checked that results have not been unduly influenced by the choice of cluster. In particular, since a cluster centred on a tetrahedral site will usually favour that site artificially, we have obtained results for clusters centred on more than one site. Thirdly, we have considered the possibility of molecular hydrogen. For hydrogen, as opposed to muons, in diamond or silicon, this species is probably the most important, whether in isolation or trapped by some other defect or impurity.

Our theoretical approach is best suited to energy surfaces, so we shall not comment in detail on the relation of our work to the calculations of hyperfine constraints by Das et al (1983), Katayama-Yoshida and Shindo (1983) and others. Regarding energy surfaces, we make three remarks. Firstly, Das et al (1983) deduce the tetrahedral site is stable in diamond. Some of our calculations on smaller clusters centred on the tetrahedral site (and hence corresponding more closely to the cluster considered by Das et al (1983)) showed this. Our extended calculations suggest that the muon is not localised by the weak minimum, although the small energy differences involved and the fact that the $\mathrm{H}^{+}$ and $\mathrm{H}^{0}$ surfaces were not resolved suggest that further investigation is needed. Secondly, Rodriguez et al (1979) found what might be an asymmetric energy minimum along the back bond for silicon. On occasions we found similar features; however, these always proved to be saddle-points, i.e. only minima when one restricted attention to energies along a specific symmetry axis. Thirdly, Pantelides et al (1983) suggested that, as a rather general rule, energy surfaces along the path linking hexagonal and tetrahedral sites should be very flat. Qualitatively, we agree, although the energy variation we predict is one that cannot be ignored in serious quantitative analysis.

\section{Discussion}

The extraordinary feature of our results is how dissimilar the three systems $\left(C: \mathrm{H}^{0}\right.$; $\mathrm{Si}: \mathrm{H}^{+} ; \mathrm{S}: \mathrm{H}^{0}$ ) are. The only similarities seem to be that the tetrahedral site is not lower in energy than other possibilities (contrary to the assumptions of some other work), and that the muon or muonium does not appear to be localised at any site in perfect diamond or silicon once one has included zero-point motion. We would suggest that either a 
tunnelling model or some other model invoking transient states must be adopted, remembering that since the lifetime of the muon is so short $\left(2.2 \times 10^{-6} \mathrm{~s}\right)$, experiments may be picking up metastable or transient states of the muon rather than its ideal stable ground state. Estle (1981) has suggested that transient lattice defects may be a component of anomalous muonium, and this is consistent with our conclusions.

Hydrogen appears to have the molecular form in silicon but is probably atomic in diamond, at least as an isolated impurity. The molecular form explains the absence of electrical, optical and infrared activity of hydrogen in silicon, where even estimates of its abundance are infrequent and confused. We have no way of telling whether the experimental discrepancies in mobility data indicate different forms of hydrogen diffusing $-\mathrm{H}_{2}$ and $\mathrm{H}^{0}$ perhaps—or whether they merely reflect experimental difficulties. In diamond, the large amounts of hydrogen are similarly invisible. We cannot explain this by simple molecule formation, except perhaps in association with impurities. However, whatever the stable form for atomic hydrogen in diamonds is, it will be extremely mobile, and this does seem to be confirmed by experiment (see the Appendix).

We now return to Estle's analysis of the muon data, which invoked a number of useful and sensible working assumptions. Since it seems likely now that at least one of these must be violated, we shall outline the possibilities by considering four basic questions.

(i) Is the same model appropriate for diamond, $\mathrm{Si}$ and $\mathrm{Ge}$ ? The problem case is diamond, where the wide band gap and the properties of the basic defects are quantitatively (and sometimes qualitatively) different. Thus the $\mathrm{N}$ donor in diamond has trigonal, not cubic, symmetry. Likewise, in Ge there are smaller isovalent impurities $(\mathrm{Si}, \mathrm{C})$, as there are in $\mathrm{Si}(\mathrm{C})$, whereas diamond, being in the first row of the periodic table, has no comparable smaller impurity. Experimentally one notes that the hyperfine constants of normal muonium do not change monotonically along the sequence (diamond, $\mathrm{Si}, \mathrm{Ge}$ ). For the hydrogen data (as opposed to muon data) it is clear the molecular form $\mathrm{H}_{2}$ should be considered.

(ii) Are impurities or defects involved? The general impression emerging is that simple donors and acceptors have only a secondary influence and are not immediately involved (Clawson et al 1981, Boekema et al 1981). However, one cannot rule out 'spur' defects, i.e. the damage (which may recover rapidly) that the muon itself causes near the end of its track (Stoneham 1979, Bucci et al 1981). The problem here is that displacement energies are quite large: one does not get vacancies and interstitials produced by recombination alone. Whether the chemical involvement of the muon can make a significant difference remains to be seen.

(iii) Is the muon tunnelling rapidly, either around a defect or impurity, or in a selftrapped local tunnelling state (Stoneham 1972, 1977, 1979)? Against tunnel models, Estle (1981) comments on the lack of line broadening, the lack of novel temperature dependences and the absence of complex spectra (see Blazey et al 1981). However, Haller (1981) has commented that normal muonium could be described by a tunnelling model, and any firm conclusion must be based on a detailed model with actual parameters. There is evidence that hydrogen in diamond is mobile, but that some localised states or traps exist at interfaces.

(iv) Should one think of muonium as $\left[\mu^{+} e\right]$, as some excited state $\left(\left[\tilde{\mu}^{+} e\right]\right.$ or $\left.\left[\mu^{+} e^{\sim}\right]\right)$, as some bound-exciton state $\left(\left[\mu^{+} \mathrm{e}\right.\right.$ eh] etc (Stoneham 1979))? The important points are (i) that electronic decay times may be comparable with the muon lifetime, (ii) that isotropic hyperfine interactions measure the unpaired spin density at the muon of all 
electrons that contribute (a point exploited in Haller's ideas), so a bound exciton could well have an observable effect, and (iii) that electrons in very extended orbits probably contribute little to the potential energy surfaces.

\section{Acknowledgments}

We are especially grateful to the many experimenters for their comments on and insights into the data, and notably the comments on the implications and credibility of the data. Among those we wish to thank are Dr K Blazey, Professor C Boekema, Professor J W Corbett, Professor T P Das, Professor T L Estle, Dr A H Harker and Professor J P F Sellschop. One of us (AM) is grateful for De Beers' support for the work on diamond.

\section{Appendix. Experiment and $\mathrm{H}$ and diamond and $\mathrm{Si}$}

Rather than interrupt the argument in the main text with details of diverse and confusing data, we summarise the experimental data here.

Chemical analysis by various methods. Recent work on diamond has exploited the nuclear reaction $\mathrm{H}\left({ }^{19} \mathrm{~F}, \alpha \gamma\right){ }^{16} \mathrm{O}$ and the infrared absorption at $3100 \mathrm{~cm}^{-1}$ associated with $\mathrm{C}-\mathrm{H}$. The conclusions are dramatic. Firstly, the concentration of $\mathrm{H}$ is large (Sellschop 1979 , Sellschop et al 1979), roughly 0.1 to $1 \%$ atomic. Secondly, even this is enhanced by a factor of up to 10 near the surface (Sellschop et al 1979). Thirdly, there is no correlation of total hydrogen with the $\mathrm{C}-\mathrm{H}$ infrared absorption, either spatially within a single diamond (Lang et al 1981), or among a group of diamonds (Annegarn et al 1979). Nor is there correlation with other bands attributed to hydrogenic defects; likewise, there is too little oxygen for water to be responsible (Sellschop et al 1979). Various suggestions are made of separate hydrogen-rich phases, such as sub-microscopic magma droplets (Sellschop et al 1979). In type I diamonds (but not type II), and especially in cloudy specimens, Woods and Collins (1983) saw lines they attribute to hydrogen at internal surfaces or cavities in the form of $\mathrm{CH}_{2}$ radicals, although hydrogen in this form does not account for the abundance of hydrogen in the bulk crystal. A recent review of the impurity levels in natural diamond, including hydrogen, is given by Bibby (1982).

In crystalline silicon, where (unlike in amorphous $\mathrm{Si}$ ) hydrogen is conspicuous by its absence, channelling analyses (Picraux and Vook 1978) of implanted deuterium have been successful in identifying $\mathrm{H}$ displaced off the tetrahedral site towards a $\langle 111\rangle$ backbond direction. It is not clear if a lattice defect is involved or not.

Diffusion. In diamond, heating experiments (Sellschop et al 1979) show that hydrogen has a high mobility, even moving significantly at room temperature under irradiation. In silicon, the large differences between the only two available estimates raise doubts about precisely what was seen (Van Wieringen and Warmolitz (1956) give $\left(9.4 \times 10^{-3}\right.$ $\left.\mathrm{cm}^{2} \mathrm{~s}^{-1}\right) \exp \left(-0.48 \mathrm{eV} k T^{-1}\right)$ and Ichimiya and Furyichi (1968) give $\left(4.2 \times 10^{-5} \mathrm{~cm}^{2}\right.$ $\left.\left.\mathrm{s}^{-1}\right) \exp \left(-0.56 \mathrm{eV} k T^{-1}\right)\right)$.

Other methods. Photoconductivity experiments under stress in the very purest Ge grown in a hydrogen atmosphere (Joos et al 1980) show an abrupt transition consistent with dynamic tunnelling of hydrogen associated with an oxygen centre. For diamond, Pepper (1981, 1982) and Wadawski et al (1982) have collected data on near-surface hydrogen in diamond in experiments ranging from electron energy loss to diamond/metal friction. Cardona (1983) reviews vibrational spectra of $\mathrm{H}$ in amorphous and crystalline $\mathrm{Si}$ and $\mathrm{Ge}$. 
Muon spin rotation. This technique has the major advantage of being a resonance method that monitors the behaviour of the major fraction of the muons entering the sample; it does not rely on unpaired electron spins. There are possible compensating problems: the muon is lighter than the proton, and so more prone to tunnelling, and the muon has a finite lifetime of about $2 \mu \mathrm{s}$, and so may reside in a metastable or transient state for the whole period monitored (Stoneham 1979).

Much of the literature is reviewed by Estle (1981). The main features concern the existence of two forms of muonium (i.e. $\left[\mu^{+} e\right]$ in some state). Normal muonium $(\mathrm{Mu})$ has full tetrahedral symmetry, with an isotropic hyperfine constant a significant fraction of the vacuum value ( $83 \%$ for diamond, $45 \%$ for $\mathrm{Si}, 53 \%$ for $\mathrm{Ge}$ ). Anomalous muonium $\left(\mathrm{Mu}^{*}\right)$, by contrast, has a highly anisotropic trigonal hyperfine structure, consistent with mainly p-like electronic structure. Other papers discuss the effects of temperature (Blazey et al 1981) and doping (Clawson et al 1981); results are not always consistent, which may reflect the indirect consequences of Fermi level shifts. However, there is evidence for transitions between $\mathrm{Mu}$ and $\mathrm{Mu}^{*}$ (see, e.g., Patterson 1984).

Pion experiments. The $\mu^{+}$produced by $\pi^{+}$decay shows a channelling pattern (Maier and Seeger 1983), which can be used to identify the pion site. Data (see $\S 1$ ) are available only for $\mathrm{Ge}$.

Positron experiments. Two important features are the positron lifetime, which roughly monitors the electron density at the positron site, and the question of impurity and spur-reaction effects. Recent work shows a monotonic trend in lifetime with atomic number (diamond, $\mathrm{Si}, \mathrm{Ge}$ ) and suggests no significant impurity effects.

\section{References}

Altarelli M and Hsu W Y 1979 Phys. Rev. Lett. 431346

Annegarn H J, Madiba C C P, Sellschop J P F, Burgemeister E A and Seal M 1979 Abstracts of the Cambridge Diamond Conference unpublished, $\mathrm{p} 45$

Bibby D M 1982 Chem. Phys. Carbon 181

Blazey K W, Brown J A, Cooke D W, Dodds S A, Estle T L, Haffner R H, Leon M and Vanderwater D A 1981 Hyperfine Interactions 8381

Boekema C, Holzschuh E, Kundig W, Meier P F, Patterson B D, Reichart W and Rjegg K 1981 Hyperfine Interactions 8401

Bucci C, de Penzi R, Guidi G, Podini P, Tedeschi R and Norlin L O 1981 Hyperfine Interactions 8385

Cardona M 1983 Phys. Status Solidi b 118463

Clawson C W, Crowe K M, Rosenblum S S and Brewer J H 19818397

Coker A, Das T P and Glodeneau A 1980 Bull. Am. Phys. Soc. 25204

Dannefaer S 1982 J. Phys. C: Solid State Phys. 15599

Das T P et al 1983 Hyperfine Interactions in press

Estle T L 1981 Hyperfine Interactions 8365

Haller E E 1981 Hyperfine Interactions 8370

Harker A H and Larkins F P 1979 J. Phys. C: Solid State Phys. 122487

Harker A H and Lyon S B 1979 Harwell Report AERE R.8598

Herbert D C and Inkson J 1977 J. Phys. C: Solid State Phys. 10 L695

Ichimiya T and Furuichi A 1968 Int. J. Appl. Rad. Isotopes 19573

Joos B, Haller E E and Fulica L M 1980 Phys. Rev. B 22833

Katayama-Yoshida H and Shindo K 1983 Phys. Rev. Lett. 51207

Lang A R, Madiba C C P, Sellschop J P F and Annegarn H J 1981 Abstracts of the Reading Diamond Conference unpublished, $\mathrm{p} 18$

Maier K and Seeger A 1983 Proc. Int. Conf. Electronic Structure of Hydrogen in Metals 1983 ed. P Jena and C Satterthwaite (New York: Plenum) p 601

1983 Hyperfine Interactions in press 
Mainwood A 1978 J. Phys. C: Solid State Phys. 112703

1979 J. Phys. C: Solid State Phys. 122543

Mainwood A and Stoneham A M 1983 Physica B 116101

Mainwood A, Stoneham A M and Larkins F P 1978 Solid State Electron. 211431

Masri P M, Stoneham A M and Harker A H 1983 J. Phys. C: Solid State Phys. 16 L613

Pantelides S T, Ivanov I, Scheffler M and Vigneron J P 1983 Physica B + C 11618

Patterson B D 1984 Muon Spin Research ed. J Chappert and R Grynszpan (Amsterdam: North-Holland) in press

Pepper S V 1981 Appl. Phys. Lett. 38344

1982 J. Vac. Sci. Technol 20 213, 643

Pickett W E and Cohen M L 1980 Phys. Rev. B 205050 (erratum Phys. Rev. B 22 3135)

Picraux S and Vook F L 1978 Phys. Rev. B 182066

Pople J A and Beveridge D L 1970 Approximate Molecular Orbital Theory (New York: McGraw-Hill)

Rodriguez C O, Jaros M and Brand S 1979 Solid State Commun. 3143

Sahoo N, Mishra S K, Mishra K C, Coker A, Das T P, Mitra C K, Snyder L C and Glodeneau A 1983 Phys. Rev. Lett. $\mathbf{5 0} 913$

Sellschop J P F, Madiba C C P and Annegarn H J 1979 Abstracts of the Cambridge Diamond Conference unpublished, $\mathrm{p} 43$

Singh V A, Corbett J W, Weigel C and Roth L M 1978 Phys. Lett. 65A 261

Singh V A, Weigel C, Corbett J W and Roth L M 1977 Phys. Status Solidi b 81637

Steeples K, Dearnaley G and Stoneham A M 1980 Appl. Phys. Lett. 36981

Stoneham A M 1972 Ber. Bunsenges. Phys. Chem. 76816

- 1977 J. Nucl. Mater. $69 / 70109$ 1979 Hyperfine Interactions 6211

Waclawski B J, Pierce D T, Swanson N and Celotta R J 1982 J. Vac. Sci. Technol. 21368

Wang J S and Kittel C 1973 Phys. Rev. B 7713

van Wieringen A and Warmolitz N 1956 Physica 22849

Woods G S and Collins A T 1983 J. Phys. Chem. Solids 44471 